\title{
Impact de la réduction de péremption des concentrés plaquettaires de 7 à 5 jours sur la gestion des stocks d'une banque de sang
}

\author{
S. Herens ${ }^{a}$, A. Gothot ${ }^{a}$, M. Monfort ${ }^{a}$
}

${ }^{a}$ Centre Hospitalier Universitaire de Liège, Laboratoire d'Immuno-Hématologie - Banque de Sang, Liège, Belgique

Suite à la modification de l'Arrêté Royal du 04/04/94 publiée au Moniteur belge le 06/02/18 réduisant la durée de validité des concentrés plaquettaires $(C P)$ de 7 à 5 jours, une réorganisation a dû être implémentée dans la plupart des banques de sang (BDS), dont l'approvisionnement dépend du Service Francophone du Sang (SFS), afin de limiter les péremptions et de parer à la moindre disponibilité des produits.

\section{Impact}

Si cette nouvelle législation a permis de délivrer des CP plus frais $(2,9 \mathrm{j}$ vs $3,6 \mathrm{j})$, elle a engendré un impact non négligeable à la fois sur la disponibilité des produits $(8.9 \%$ des commandes ne pouvant être honorées) et sur la gestion des péremptions. L'analyse des données des CP en 2018 par rapport à la même période en 2017 a en effet montré un taux de péremption multiplié par 2 :

\begin{tabular}{|l|c|c|}
\hline & $\begin{array}{c}\mathbf{2 0 1 7} \\
(\mathbf{1 2 / 0 2} \rightarrow \mathbf{3 1} / \mathbf{0 7})\end{array}$ & $\begin{array}{c}\mathbf{2 0 1 8} \\
(\mathbf{1 2} / \mathbf{0 2} \rightarrow \mathbf{3 1} / \mathbf{0 7})\end{array}$ \\
\hline Age moyen à la réception & $2.2 \mathrm{j}$ (SD 1.04) & $2.1 \mathrm{j}$ (SD 0.99) \\
\hline Age moyen à la délivrance & $3.6 \mathrm{j}$ (SD 1.42) & $2.9 \mathrm{j}$ (SD 1.11) \\
\hline Age médian à la délivrance & $4 \mathrm{j}$ & $3 \mathrm{j}$ \\
\hline Taux CP délivrés $>5 \mathrm{j}$ & $\begin{array}{c}9.16 \% \\
(8.39 \% \mathrm{CUP},\end{array}$ & $0 \%$ \\
\hline Taux de péremption (TP) & $\begin{array}{c}0.77 \% \text { POOL) } \\
1.33 \%\end{array}$ & $2.5 \%$ \\
\hline Coût lié à la péremption & $15363.04 €$ & $27982.68 €$ \\
\hline
\end{tabular}

\begin{tabular}{|c|c|}
\hline AVANTAGES & INCONVENIENTS \\
\hline $\begin{array}{c}\searrow \text { âge à la délivrance : } \\
\lambda \text { transfusion de CP frais }\end{array}$ & $\lambda$ taux de péremption \\
\hline & $\frac{\lambda \text { moyens logistiques }}{\lambda \text { coûts }}$ \\
\hline & $\frac{\text { Pas de } \searrow \text { d'âge à la réception }}{}$ \\
\hline
\end{tabular}

Age à la délivrance des $\mathrm{CP}$

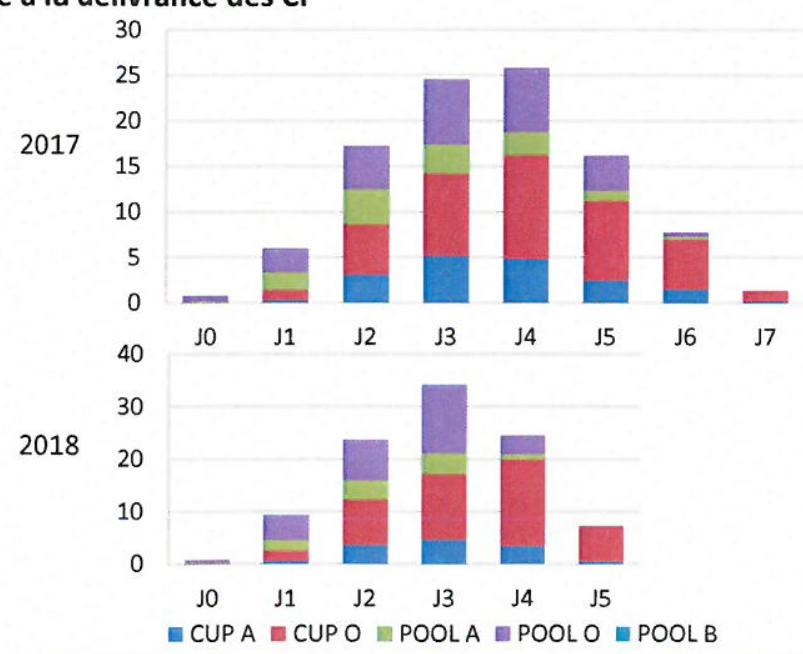

\section{Adaptation organisationnelle}

Mesures mises en place dans la banque de sang et au SFS :

- modification des habitudes transfusionnelles : rappel des seuils transfusionnels, meilleure planification des transfusions en fonction des livraisons des CP

- optimalisation de la gestion des stocks : surveillance continue, meilleure anticipation

- extension des plages horaires de prélèvement au SFS (prélèvement le samedi)

- modification des fréquences et horaires de livraison

$\Rightarrow$ réduction du taux de péremption $2.4 \% \rightarrow 1.6 \%$ après l'implémentation des différentes mesures

$\Rightarrow$ réduction du nombre de commandes de $\mathrm{CP}$ non honorées $10.3 \% \rightarrow 8.2 \%$

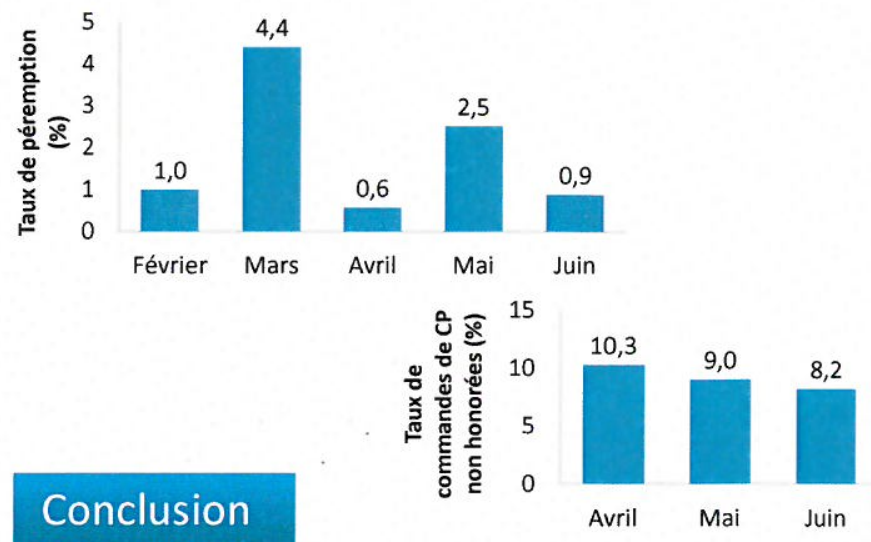

Après une période d'adaptation difficile, des mesures ont été mises en place à la BDS et au SFS. Cette collaboration et ces actions correctives ont eu un impact positif, permettant à la fois de diminuer le nombre de commandes non honorées faute de CP disponibles tout en permettant une réduction des TP de $30 \%$. Cependant, la question des bases scientifiques à cette réduction de délai se pose, aucune étude n'ayant démontré clairement l'impact positif de la transfusion de CP frais chez les patients non hématologiques ${ }^{1}$. La question d'un allongement à 7 jours en fonction de l'indication devrait être étudiée afin de pouvoir répondre à toutes les commandes.

\section{Contact}

Sophie Herens

S.Herens@chuliege.be

\section{Référence}

${ }^{1}$ Aubron, Cécile, et Al. Platelet storage duration and its clinical and transfusion outcomes : a systematic review. Critical care. 2018, 22:185 